\title{
Review of: "Nanoengineering of eco-friendly silver nanoparticles using five different plant extracts and development of cost-effective phenol nanosensor"
}

\author{
Mehran Alavi
}

Potential competing interests: The author(s) declared that no potential competing interests exist.

Please consider the below comments to improve the manuscript titled "Nanoengineering of eco-friendly silver nanoparticles using five different plant extracts and development of cost-effective phenol nanosensor":

1. In the introduction section, fourth paragraph, both primary and secondary metabolites can contribute to metal NP synthesis, therefore correct the sentence "for the synthesis of NPs, the primary plant compounds......",

2. Where is related reference for this claim "a relatively fast reduction of metal ions, which easily occurs in solution and leads to a high density of stable silver nanoparticles."? Green synthesis by plant extracts may be resulted in unstable AgNPs, which authors should address this disadvantage. In addition, for fast reduction property of green synthesis by plants, references of comparative studies about various synthesis methods are required. In the best way, I think that authors can provide one table about advantages and disadvantages of AgNP or metal NP synthesis by different methods.

3. What are your reasons for selection five plant species of Basil, Geranium, Eucalyptus, Melia, and Ruta. Please write complete name of plant species (genus +species), moreover why did you leaf extract? Flowers and stems also have phenolic compounds.

4. In the section of results, Figure 2, don't use wavelength $(\mathrm{nm})$ for horizontal axis for FTIR spectra, wavenumber $(\mathrm{cm}-1)$ is correct.

5. Authors should add the results of polydispersity index (PDI) for five NPs.

6. In figures $5 A-D$, original SEM images with related scale bar are needed. The quality of figure $5 \mathrm{~F}$ is not suitable.

7. Discussion and collusion sections are weak and should be extended.

8. The manuscript would benefit from English grammar editing by a native person. 